\title{
Binding Affinity, Cellular Uptake, and Subsequent Intracellular Trafficking of the Nano-Gene Vector P123-PEI-R13
}

\author{
Yaguang Zhang, Hongmei Shu, Jing Hu, Min Zhang, Junweng Wu, Kehai Liu, and Qing Zhu \\ Department of Biopharmaceutics, College of Food Science and Technology, Shanghai Ocean University, Shanghai 201306, China \\ Correspondence should be addressed to Kehai Liu; khliu@shou.edu.cn
}

Received 26 July 2016; Revised 8 September 2016; Accepted 4 October 2016

Academic Editor: Liqian Gao

Copyright (c) 2016 Yaguang Zhang et al. This is an open access article distributed under the Creative Commons Attribution License, which permits unrestricted use, distribution, and reproduction in any medium, provided the original work is properly cited.

\begin{abstract}
A nano-gene vector PEI-P123-R13 was synthesized by cross-linking low molecular weight PEI with P123 and further coupling bifunctional peptide R13 to the polymer for targeting tumor and increasing cellular uptake. The binding assessment of R13 to $\alpha \mathrm{v} \beta 3$ positive cells was performed by HRP labeling. The internalization pathways of P123-PEI-R13/DNA complexes were investigated based on the effect of specific endocytic inhibitors on transfection efficiency. The mechanism of intracellular trafficking was investigated based on the effect of endosome-lysosome acidification inhibitors, cytoskeleton, and dynein inhibitors on transfection efficiency. The results indicated that the bifunctional peptide R13 had the ability of binding to $\alpha \mathrm{v} \beta 3$ positive cells in vitro. The modification of P123-PEI-R13 with R13 made it display new property of internalization. P123-PEI-R13/DNA complexes were conducted simultaneously via clathrin-mediated endocytosis, caveolin-mediated endocytosis, macropinocytosis, and possible energy-independent route. After internalization, P123-PEI-R13/DNA complexes could escape from the endosome-lysosome system because of its acidification and further took microtubule as the track and dynein as the dynamic source to be transported toward the microtubule $(+)$ end, to wit nucleus, under the action of microfilament, and with the aid of intermediate filament.
\end{abstract}

\section{Introduction}

Cancer is the main reason of death worldwide, while gene therapy is the most potential way to eradicate cancer. A high efficiency and low toxicity gene vector plays a key role in gene therapy. Nonviral gene vectors, especially polyethyleneimine (PEI), have recently attracted remarkable interests. A targeting vector P123-PEI-R13 was once successfully synthesized. At first we synthesized a kind of high molecular weight PEI derivate (P123-PEI) by cross-linking low molecular weight PEI with Pluronic P123. Integrin $\alpha \mathrm{v} \beta 3$ can be highly expressed on cancer cells, and RGD is the $\alpha \mathrm{v} \beta 3$-targeting peptide. Cell-penetrating peptides can directly carry macromolecule substance to pass through the cell membrane. In order to improve cell selection and increase cellular uptake, we coupled $\alpha \mathrm{v} \beta 3$-targeting peptide RGDC with the cell-penetrating peptide TAT to obtain a bifunctional peptide RGDCTAT (named R13) and then used R13 to modify the PEI derivate P123-PEI. Thus a new polymeric gene vector (P123PEI-R13) was prepared. The purpose of this study was to solve the efficiency-versus-cytotoxicity and tumor-targeting problems of PEI as a nonviral gene delivery vector. The new nonviral gene vector P123-PEI-R13 could reduce cytotoxicity of PEI under the circumstance that PEI showed high transfection efficiency, increase the cell selection, and then improve the curative effect of gene therapy. This study could find an available way to gene therapy on cancer [1]. However, the binding affinity, cellular uptake, and subsequent intracellular trafficking of this vector should be clarified.

For gene therapy, an appropriate gene vector is necessary for its efficient binding to cells, cellular uptake, and intracellular trafficking. Binding to the target cells is an essential precondition for cell transfection. The internalization of macromolecules is achieved through endocytosis. Endocytosis is divided into phagocytosis and pinocytosis, in which phagocytosis exists in specialized cells while pinocytosis is involved in all kinds of cells. During the endocytosis, plasma membrane is internalized into cells to form a vesicle firstly and then enter the endolysosomal environment by fusing with endosomes [2]. Phagocytosis can be classified into the following: clathrin-mediated endocytosis, caveolin-mediated 
endocytosis, macropinocytosis, and clathrin- and caveolaeindependent endocytosis [3]. After the complex is taken up by cells via endocytosis, the further intracellular transport from endosome to the nucleus appears to be particularly important. Endosome-lysosome system is the main pathway. As a highway to transport the cargoes, the cytoskeleton is made of three kinds of protein filaments: microfilaments, intermediate filaments, and microtubules [4].

Chemical inhibitors have an enormous impact on the intracellular components and structures, and the major intracellular pathways of macromolecules are usually distinguished on the basis of their differential sensitivity to chemical inhibitors. In this study, inhibitors were applied to determine the internalization mode and intracellular routing of P123-PEI-R13/DNA complexes. Chlorpromazine hydrochloride (CPZ), glucose, and C9 (competitive peptide RGD, CYGGRGDTP) were used as inhibitors of clathrin-mediated endocytosis. CPZ was said to inhibit clathrin-mediated endocytosis by translocating clathrin and adaptin from membrane to vesicles [5]. Glucose might lead to the shedding of clathrin [6]. The inhibition of C9 was competitive. It blocked the combination between P123-PEI-R13/DNA and adaptin, which regulated clathrin-mediated endocytosis [7]. Genistein was used as an inhibitor of caveolin-mediated endocytosis. It restrained the formation of intracellular vesicles [8]. 5(N,N-Dimethyl) amiloride hydrochloride (DMA) was used as an inhibitor of macropinocytosis. It could inhibit $\mathrm{Na}^{+} / \mathrm{H}^{+}$ exchange protein on the plasma membrane [9]. $\mathrm{NH}_{4} \mathrm{Cl}$ and monensin were used as inhibitors of endosome-lysosome system. They prevented the acidification of endosome and inhibited their fuse and maturation [10]. Cytochalasin B (Cyto B) caused the depolymerization of the action and inhibited the function of microfilaments [11]. Colchicine (Col) and paclitaxel (PTX) were used as inhibitors of microtubes. Col could stimulate polymerization of microtubes and PTX could inhibit mitosis [12, 13]. Having determined suitable inhibitors, the efficiency of inhibitors was assessed quantitatively in Hela cells by pGL3-Control reporter gene so as to investigate the internalization pathways and intracellular trafficking of P123-PEI-R13/DNA complexes based on the effect of specific inhibitors on transfection efficiency. Understanding the intracellular transport can provide researchers with more ideas for improving current nonviral gene vectors. This study investigated the intracellular transport of gene delivery system for cancer gene therapy with the purpose of developing an optimal prototype for tumor cell transfection.

\section{Materials and Methods}

2.1. Materials. HRP, SMCC, BSA, genistein, monensin, cytochalasin B, glucose, colchicine, paclitaxel (PTX), and 5-(N,N-dimethyl) amiloride hydrochloride (DMA) were obtained from Sigma Aldrich (St.Louis, MO). The competitive peptide RGD C9 (CYGGRGDTP), dimethylsulfoxide (DMSO), formaldehyde, $\mathrm{H}_{2} \mathrm{O}_{2}$, EDTA, $\mathrm{NH}_{4} \mathrm{Cl}$, acrylamide, and the absolute ethyl ethanol were obtained from China National Medicines Corporation Ltd. RPMI 1640 culture medium, fetal bovine serum (FBS), and trypsin were purchased from Invitrogen (Carlsbad, CA). Luciferase assay system for in vitro transfection assay and pGL3-Control vector with SC-40 promoter and enhancer encoding firefly (Photinus pyralis) luciferase were obtained by Promega (Madison, WI). Chlorpromazine hydrochloride (CPZ) and sodium orthovanadate (SOV) were provided by TCI (Shanghai) Development Co., Ltd.

2.2. Preparation of P123-PEI-R13/DNA Complexes and Morphologic Observation. The charge ratio of P123-PEI-R13/ DNA was controlled by regulating weight ratio of P123-PEIR13 and DNA. DNA solution and polymer solution were mixed to form self-assembly complexes with desired w/w ratio. The complexes were allowed to stand at $4^{\circ} \mathrm{C}$ for 30 minutes before they were used in the next experiments.

The concentration of DNA was $50 \mu \mathrm{g} / \mathrm{mL}$ and the w/w ratio of the polymer to DNA was 10. A drop of the complex solution was placed on a copper grid. Transmission electron microscope (JEM 2100F; JEOL Ltd., Tokyo, Japan) was used to observe the morphological characteristics of the micelle after the sample was dried.

\subsection{Binding Affinity of R13 to Integrin Receptor}

2.3.1. Conjugation of R13 with HRP. N-Succinimidyl-4-(Nmaleimido-methyl) cyclo-hexane-carboxylate (SMCC) was used as heterobifunctional agent to combine R13 with HRP enzyme. After SMCC was dissolved in dimethyl sulfoxide solution $(3.33 \mathrm{mg} / \mathrm{mL})$ and $\mathrm{HRP}$ was dissolved in PBS arriving at a concentration of $10 \mathrm{mg} / \mathrm{mL}, 0.1 \mathrm{~mL}$ of SMCC solution was slowly added to $0.4 \mathrm{~mL}$ of HRP solution $(1: 10, \mathrm{n} / \mathrm{n})$. The reaction mixture was incubated at $25^{\circ} \mathrm{C}$ for $30 \mathrm{~min}$. The superfluous nonreacted cross-linker was removed by gel chromatography (Sephadex G-25, Pharmacia). Then $10 \mathrm{mg} / \mathrm{mL}$ R13 PBS solution was mixed with the maleimide-activated HRP solution $(1: 10, \mathrm{n} / \mathrm{n})$ and kept at $4^{\circ} \mathrm{C}$ in shade for 12 hours. Amicon Ultra-4 centrifugal filter device (Millipore) was used to remove the nonreacted R13.

2.3.2. Evaluation of Binding Affinity. As we know, the integrin $\alpha \mathrm{v} \beta 3$ receptors can be highly expressed on Hela cells and B16 cells. Two kinds of cells were seeded in RMPI 1640 medium in 6-well plates (Costar, Corning Corp, New York) and incubated for one day to $70-80 \%$ confluence. After the medium was removed, the cells were washed by PBS once. $1 \mathrm{~mL}$ of fixative solution (2\% formaldehyde and $0.05 \%$ glutaral in PBS buffer) was added to each well and the cells were fixed for 15 minutes. The cells were washed by $1 \mathrm{~mL} 0.01 \mathrm{M}$ PBS three times, treated with $3 \% \mathrm{H}_{2} \mathrm{O}_{2}$ aqueous solution, and then washed by $3 \mathrm{~mL}$ of $0.01 \mathrm{M}$ PBS three times, each time for 3 minutes. $1 \mathrm{~mL}$ of $1 \%$ BSA was added in each well to block the protein binding site. After 20 minutes, the excess liquid was blotted. The cells were randomly divided into test groups and control groups, which were cultivated with the diluted R13-HRP solutions and HRP solutions for 60 minutes at $37^{\circ} \mathrm{C}$, respectively, and then washed by $3 \mathrm{~mL} 0.01 \mathrm{M}$ PBS three times. Finally, the cells were stained with DAB and observed by microscopy (AE-31, Motic Corporation, Germany). 
2.4. Effect of Specific Endocytic Inhibitors on Transfection Efficiency. The effects of different inhibitors on the transfection efficiency of P123-PEI-R13/DNA in Hela cells were investigated using the pGL3-Control reporter gene. After being dispersed by trypsin, $3 \times 10^{4}$ cells were added to each well of a 24-well plate and cultured with RPMI 1640 medium containing $10 \%$ serum for $24 \mathrm{~h}$ to $70-80 \%$ confluence. Before transfection, the culture medium was removed and then washed by $1 \mathrm{~mL} 0.01 \mathrm{M}$ PBS three times. $400 \mu \mathrm{L}$ of serumfree RPMI 1640 medium containing different concentrations of inhibitors was added to each well and incubated for 30 minutes. Cells were incubated for $2 \mathrm{~h}$ at $37^{\circ} \mathrm{C}$ in $5 \% \mathrm{CO}_{2}$ atmosphere after $100 \mu \mathrm{L}$ of complex solution with $0.8 \mu \mathrm{g}$ pDNA at w/w ratio of 30 was appended to each well. Then the medium was replaced with $500 \mu \mathrm{L}$ of medium containing $10 \%$ FBS and cells were incubated for another $48 \mathrm{~h}$.

Luciferase was detected in accordance with Operation Manual (Promega, Madison, WI). The culture medium was removed and $200 \mu \mathrm{L}$ of cell culture lysis reagent (CCLR) was added. After $0.5 \mathrm{~h}$ shaking, the cell lysis solution was centrifuged ( $8000 \mathrm{rpm}, 5 \mathrm{~min}, 4^{\circ} \mathrm{C}$ ). $20 \mu \mathrm{L}$ of cell culture supernate was added to $100 \mu \mathrm{L}$ of the luciferin substrate before luciferase activity was examined immediately by a luminometer (Turner Designs Luminometer model TD20/20; Promega, USA). The content of protein was detected by BCA protein assay kit (Pierce, Rockford, IL). The relative light unit (RLU) against the corresponding protein contents was applied to calculate the transfection efficiency for pGL3Control. Six replicates were counted for each sample.

2.5. Effect of Incubation Time and Temperature on Transfection Efficiency. The effect of incubation temperature and time on the transfection efficiency of P123-PEI-R13/DNA complexes was also investigated in Hela cells using the pGL3-Control reporter gene. The cells were cultured according to 2.4. After the culture medium was removed, cells were incubated with $400 \mu \mathrm{L}$ of serum-free RPMI 1640 medium and $100 \mu \mathrm{L}$ of complex solution with $0.8 \mu \mathrm{g}$ pDNA at w/w ratio of 30 at $4^{\circ} \mathrm{C}$ and $37^{\circ} \mathrm{C}$, respectively. Then the culture medium was removed at different time point and the cells were washed by PBS once. A fresh medium containing 10\% FBS was added and cells were further incubated for 48 hours. Luciferase activity was measured according to Section 2.4.

\subsection{Effect of Endosome-Lysosome Acidification Inhibitors,} Cytoskeleton, and Dynein Inhibitors on Transfection Efficiency. The effects of endosome-lysosome acidification inhibitors, cytoskeleton, and dynein inhibitors on the transfection efficiency of P123-PEI-R13/DNA in Hela cells were investigated. All the experiments using the pGL3-Control as reporter gene were performed according to Section 2.4.

2.7. Statistical Analysis. Data was showed in the form of the mean \pm standard deviation. Assigning the value of significant level as 0.05 , two-sample Student's $t$-tests and analysis of variance were used to determine statistically significant differences between these groups.

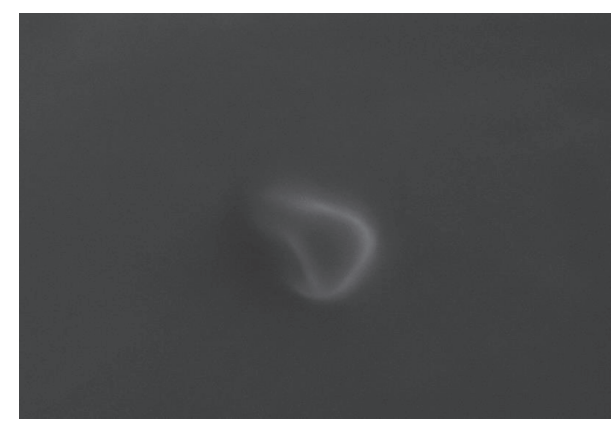

FIGURE 1: Transmission electron micrograph of P123-PEI-R13/DNA complexes.

\section{Results and Discussion}

3.1. Morphological Characteristics of P123-PEI-R13/DNA Complexes. From Figure 1, the P123-PEI-R13 polymer could condense pDNA into nanoparticles with spherical structure. The particle size and electric potential of P123-PEI-R13/ DNA complex under different mass ratios were measured previously. The particle sizes of the complexes with range from 100 to $400 \mathrm{~nm}$ were inversely proportional to its w/w ratios, which was suitable for cell transfection. As the w/w ratio of cationic polymer increases, the zeta potential of the complex also increases. An appropriate amount of positive charges helps to bind successfully to cell membranes with negative charges, but too high concentration of cationic charges will be toxic to cells. The zeta potential of P123-PEIR13/DNA complexes was around 5-40 mV.

3.2. Binding of R13 Coupled with HRP to $\alpha v \beta 3$ Positive Cells. PEI used as gene vector was limited due to its poor cells selection. PEI was positively charged and body cells were negatively charged, which result in that there was no binding selectivity between them. Hela cells were cocultured with HRPlabeled R13 and HRP, and staining was conducted with DAB, followed by an observation under an inverted microscope. In Figure 2, the cells in test groups were stained strongly positive, but negative in control groups. The results indicated that R13 was capable of binding to $\alpha \mathrm{v} \beta 3$ positive cells in vitro, Hela cells, and B16 cells and heralded excellent tumor targeting.

\subsection{Mechanism of Cellular Uptake of P123-PEI-R13/DNA Complexes}

3.3.1. Effects of Clathrin-Mediated Endocytosis Inhibitors on Cell Uptake of P123-PEI-R13/DNA Complexes. As shown in Figure 3, different concentration of CPZ, glucose, and C9 inhibited transfection efficiency of P123-PEI-R13/DNA complexes more or less. The inhibitive effect became more significant as the dose increased (except glucose). $20 \mu \mathrm{M}$ of CPZ showed the most notable inhibition $(>85 \%)$, followed by $250 \mathrm{ng} / \mathrm{mL}$ C9 $(>50 \%)$, which affected the transfection efficiency through competitively combination with receptors on the cell surface. The inhibition rate of $0.4 \mathrm{M}$ glucose is about $42 \%$. Clathrin-mediated endocytosis is the primarily 


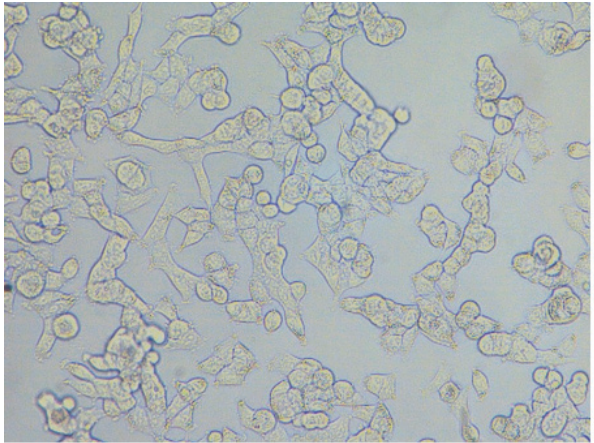

Hela cells only with HRP (200x)

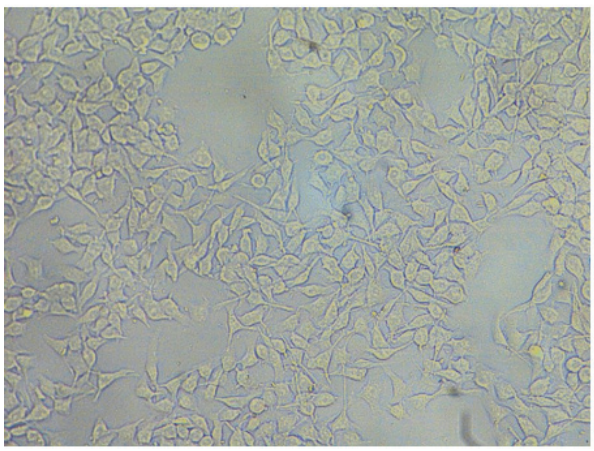

B16 cells only with HRP (200x)

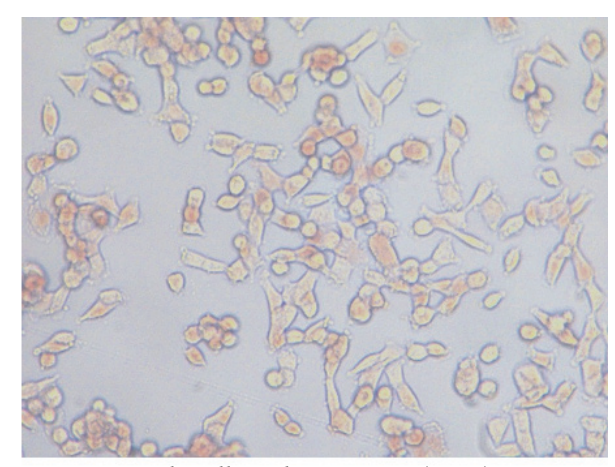

Hela cells with R13-HRP (200x)

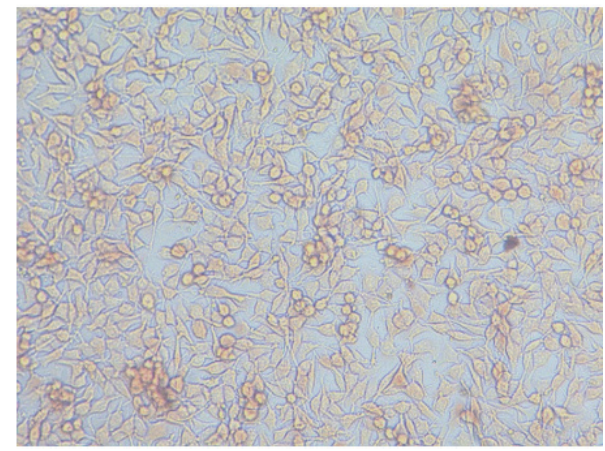

B16 cells with R13-HRP (200x)

FIgURE 2: Binding affinity of R13 to Hela cells and B16 cells.

and explicitly recognized uptake pathway of cells. Almost all mammalian cells adopt this approach for continuous endocytosis of important substances. The results demonstrated that clathrin-mediated endocytosis was one of the efficient approaches for P123-PEI-R13/DNA complexes to pass into cells.

3.3.2. Effects of Caveolin-Mediated Endocytosis Inhibitor on Cell Uptake of P123-PEI-R13/DNA Complexes. The inhibition of genistein to caveolin-mediated endocytosis was shown in Figure 4. No inhibition was found at low concentration of genistein; the inhibitive effect increased with increasing concentration. The inhibition rate is as high as $70 \%$ at the concentration of $200 \mu \mathrm{M}$. It revealed that caveolin-mediated endocytosis also caused difference in the internalization, as one of the efficient pathways employed by P123-PEI-R13/DNA complexes.

3.3.3. Effect of Macropinocytosis Inhibitor on Cell Uptake of P123-PEI-R13/DNA Complexes. The inhibition of DMA to macropinocytosis was shown in Figure 5. No inhibition was found at low concentration of DMA. Higher concentration of DMA could inhibit the transfection efficiency of P123PEI-R13/DNA complexes. The inhibitive effect was dosedependent and the inhibition rate is about $65 \%$ at the concentration of $20 \mu \mathrm{M}$. It is worth mentioning that the macropinocytosis is often neglected, and few cationic complexes get into cells through this way. However, the transfection of
P123-PEI-R13/DNA complexes was significantly inhibited in the presence of high concentration of DMA; that is to say, macropinocytosis participated in the endocytosis of P123PEI-R13/DNA complexes, which showed the different endocytosis characteristics of this complex. It may be associated to the modification of P123-PEI-R13 with cell-penetrating peptide (CPP) TAT (49-57) [14].

\subsubsection{Effect of Incubation Time and Temperature on Trans-} fection Efficiency of P123-PEI-R13/DNA Complexes. Hela cells were incubated with P123-PEI-R13/pGL3-Control complexes at $4^{\circ} \mathrm{C}$ and $37^{\circ} \mathrm{C}$, respectively, and culture medium containing compound was removed at a certain time point. The culture medium was replaced by fresh medium with 10\% FBS and cells were further incubated for 48 hours. Transfection efficiency of P123-PEI-R13/pGL3-Control complexes at different temperature and incubation time was measured, shown in Figure 6.

From Figure 6, the transfection effect of compound on cell will increase over time under $37^{\circ} \mathrm{C}$, but it did not reach a maximum after $4 \mathrm{~h}$ incubation. It also increased with time under $4^{\circ} \mathrm{C}$ but gradually leveled off after $2 \mathrm{~h}$ incubation. In addition, the complex had a higher transfection efficiency under $37^{\circ} \mathrm{C}$ than under $4^{\circ} \mathrm{C}$ at different time point, which meant that transfection of P123-PEI-R13/DNA complexes was an energy consuming process. It is worth noting that the transfection of the complex under $4^{\circ} \mathrm{C}$ was not completely restrained by the low temperature and had the time-dependent characteristic, demonstrating that the internalization of this complex 


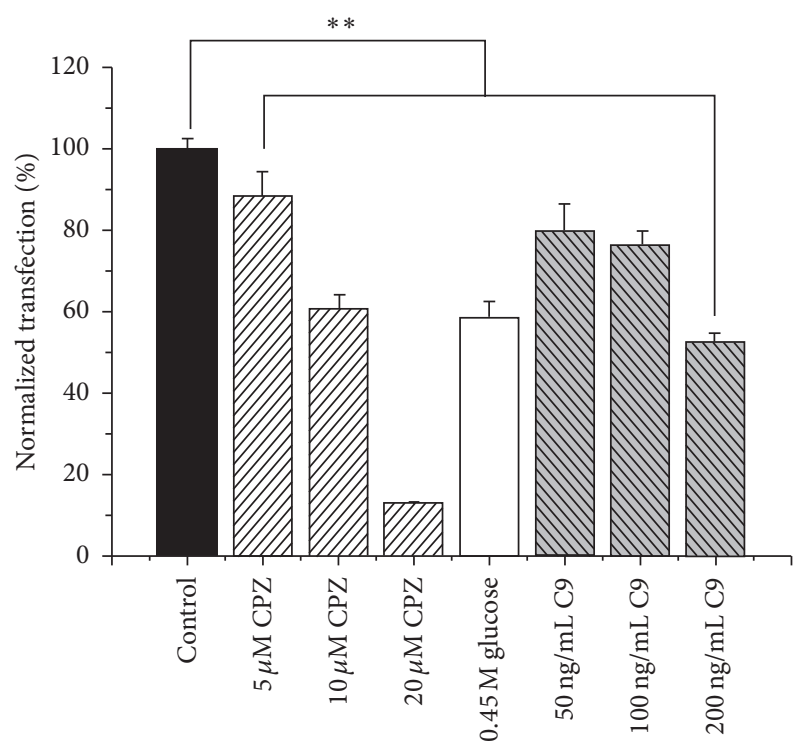

Figure 3: Effect of chlorpromazine hydrochloride (CPZ), glucose, and $\mathrm{C} 9$ on transfection efficiency of P123-PEI-R13/DNA complexes in Hela cells at w/w ratio of $30 . \mathrm{CPZ}$ and glucose were used as the inhibitors of clathrin-mediated endocytosis. C9 was the competitor of P123-PEI-R13/DNA complexes for clathrin-mediated endocytosis. The RLU/mg protein in control cells was set to $100 \%$. Each data point represents the mean \pm standard deviation $(n=6$, $\left.{ }^{* *} P<0.01\right)$.

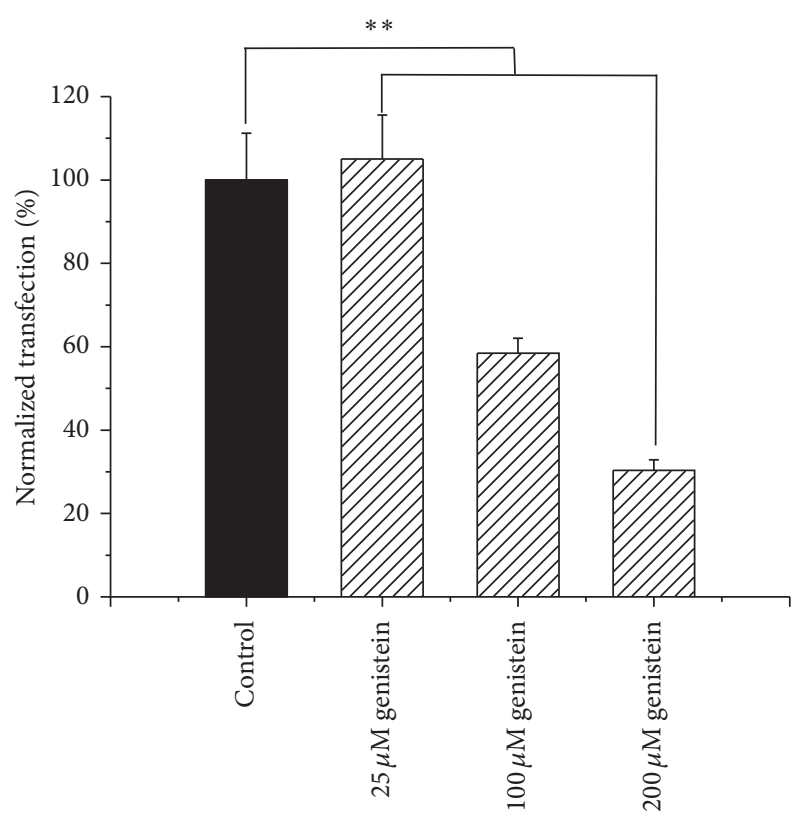

FIGURE 4: Effect of different concentration of genistein on transfection efficiency of P123-PEI-R13/DNA complexes in Hela cells at w/w ratio of 30. Genistein was used as the inhibitor of caveolin-mediated endocytosis. The RLU/mg protein in control cells was set to $100 \%$. Each data point represents the mean \pm standard deviation $(n=3$, $\left.{ }^{* *} P<0.01\right)$.

was not simply an active transport process that needed energy consumption, but there also might be other forms

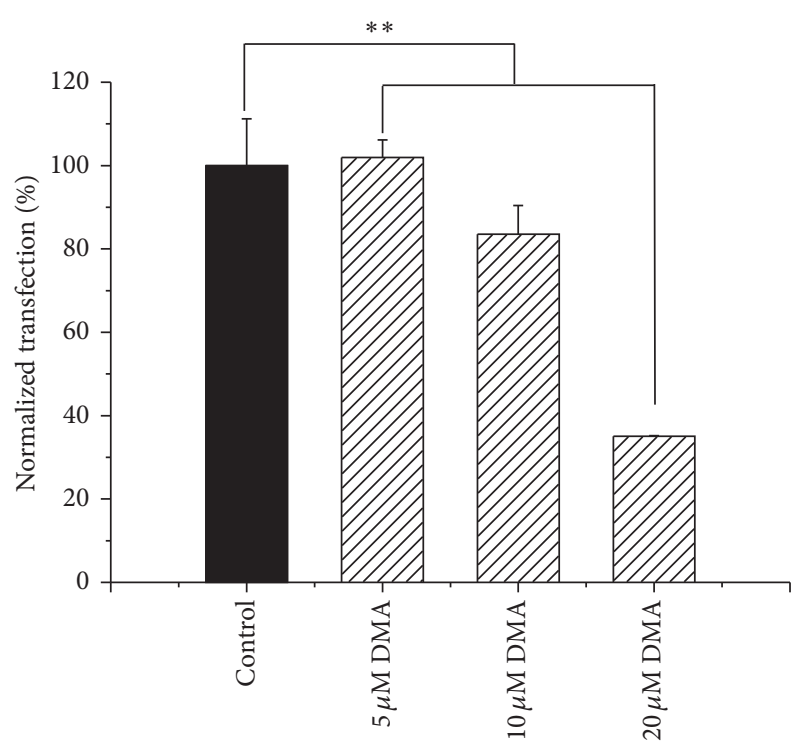

FIGURE 5: Effect of different concentration of 5-(N,N-dimethyl) amiloride hydrochloride (DMA) on transfection efficiency of P123PEI-R13/DNA complexes in Hela cells at w/w ratio of 30. DMA was used as the inhibitor of macropinocytosis. The RLU/mg protein in control cells was set to $100 \%$. Every data point represents the average value of integrated data $\left(n=3,{ }^{* *} P<0.05\right)$.

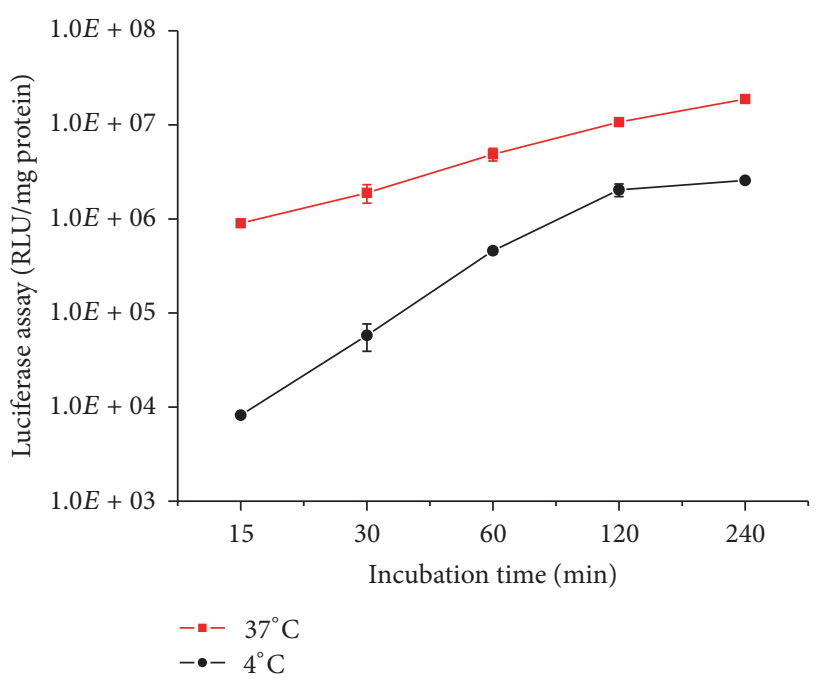

FIGURE 6: Effect of incubation time and temperature on transfection efficiency of P123-PEI-R13/DNA complexes in Hela cells at w/w ratio of 30 . Each data point represents the mean \pm standard deviation $(n=$ $5)$.

of nonendocytosis. P123-PEI-R13 was modified using the TAT (49-57) peptide. This kind of cell-penetrating peptide can carry macromolecule substance into cells directly independently of the energy consumption. Compared with the energy-dependent endocytosis, energy-independent internalization did not seem to play a dominant role, because the transfection gradually tended to balance after incubation for $2 \mathrm{~h}$ at $4^{\circ} \mathrm{C}$, and transfection ability was much weaker than that at the corresponding time point under $37^{\circ} \mathrm{C}$. 


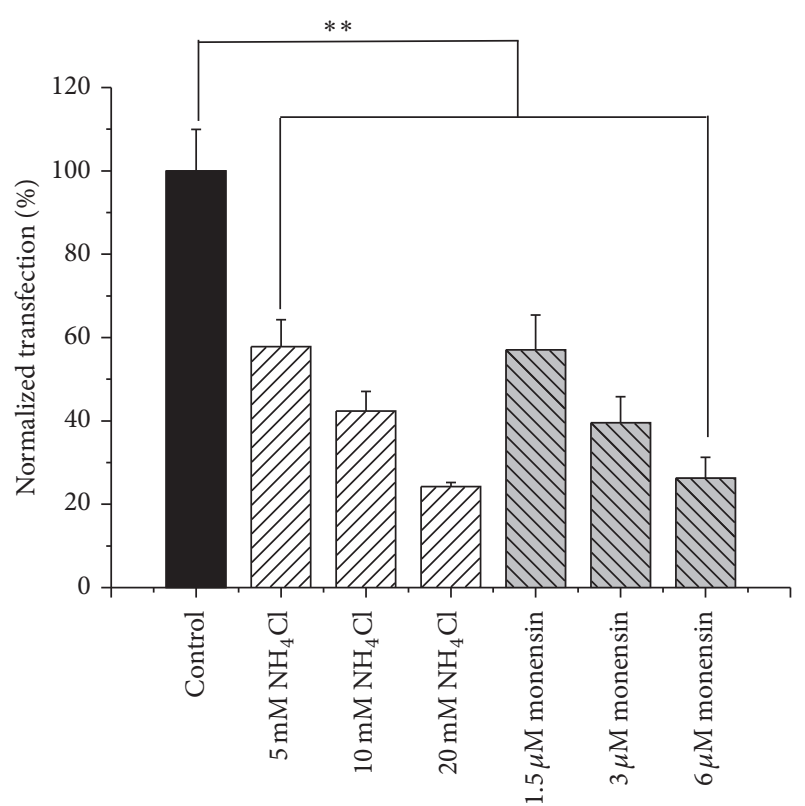

FIGURE 7: Effect of $\mathrm{NH}_{4} \mathrm{Cl}$ and monensin on transfection efficiency of P123-PEI-R13/DNA complexes in Hela cells at w/w ratio of 30. $\mathrm{NH}_{4} \mathrm{Cl}$ and monensin were used as the acidification inhibitors of endosome-lysosome system. The RLU/mg protein in control cells was set to $100 \%$. Each data point represents the mean \pm standard deviation $\left(n=6,{ }^{* *} P<0.01\right)$.

\subsection{Mechanism of Intracellular Trafficking of P123-PEI-R13/DNA Complex}

3.4.1. Effect of Acidification Inhibitors of Endosome-Lysosome System on Intracellular Transport of P123-PEI-R13/DNA Complexes. After endocytosis, the complex was delivered to lysosomes from endosomes, which was a cytosol acidification process. In Figure 7, the inhibition of $\mathrm{NH}_{4} \mathrm{Cl}$ and monensin increased as the doses were increasing; they reduced the transfection efficiency by $76 \%$ and $74 \%$, respectively, at the highest dose. These results demonstrated that part of the complex indeed underwent endosome-lysosome system in cells and acidification process is advantageous to gene transfection. It caused protonation of P123-PEI-R13 and helped the complex escape from lysosome. The acidification process of endosome-lysosome system is the basis and ensures complex transport to the nucleus [15].

\subsubsection{Effect of Cytoskeleton Inhibitors on Intracellular Trans-} port of P123-PEI-R13/DNA Complexes. As important roles in cellular activities, microtubules function as routes along which organelles can move. The assembly of microtubules is a dynamic equilibrium process of polymerizationdepolymerization. Col could cause depolymerization of microtubules, but PTX stimulated excessive polymerization. They both disrupted the balance of dynamic equilibrium process [16]. In Figure 8, $50 \mathrm{mM}$ of PTX and $20 \mu \mathrm{g} / \mathrm{mL}$ of Col reduced transfection efficiency by $50 \%$ and $80 \%$, respectively. The results revealed that the dynamic equilibrium process of microtubules was necessary for the intracellular trafficking of

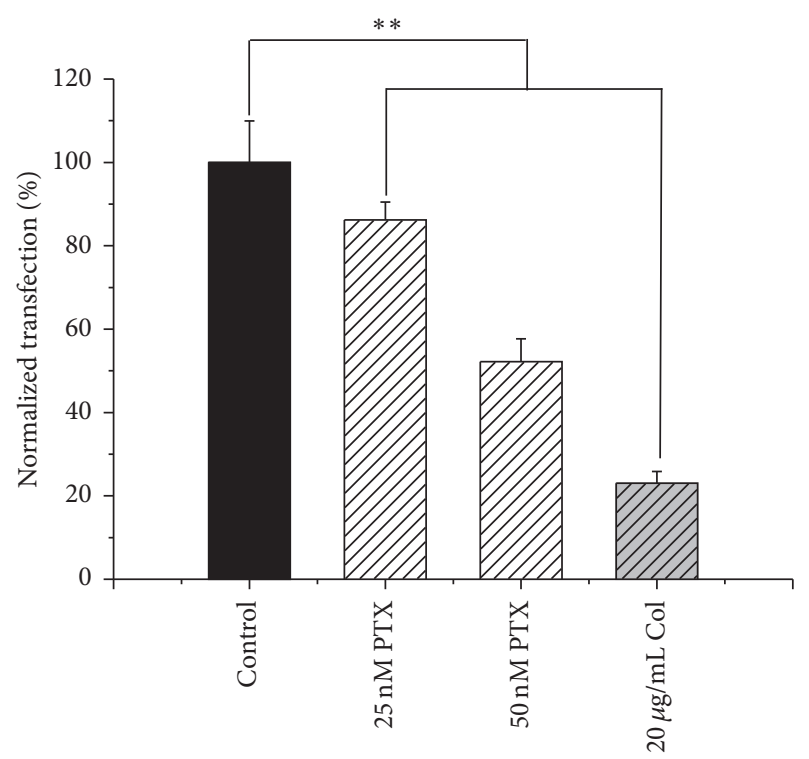

Figure 8: Effect of paclitaxel (PTX) and colchicine (Col) on transfection efficiency of P123-PEI-R13/DNA complexes in Hela cells at w/w ratio of 30 . PTX and Col were used as the inhibitors of microtubule. The RLU/mg protein in control cells was set to $100 \%$. Each data point represents the mean \pm standard deviation $(n=3$, $\left.{ }^{* *} P<0.01\right)$.

P123-PEI-R13/DNA complexes. The polymerization process stimulated the complexes tracking toward the microtubule $(-)$ end, to wit nucleus, and depolymerization process stimulated the complexes getting into nucleus through mitosis. Once the balance was broken, gene transfection efficiency would decrease.

Microfilaments are the thinnest filaments of the cytoskeleton and they function in cell movement and generate locomotion in cells. Cyto B can interrupt cell activities as it binds with the $(+)$ end of microfilaments, resulting in the depolymerization of actin filaments and the disappearance of F-actin skeleton [17]. In Figure 9, the transfection efficiency of Cyto B-treated cells was reduced considerably. The transfection process almost ground to a halt at the concentration of $18 \mu \mathrm{M}$. This result proved that microfilaments involved in the intracellular trafficking of P123-PEI-R13/DNA complexes in cells, intimately associated with cytokinesis in mitosis telophase.

The intermediate filaments have various functions during the cellular life cycle. One end of an intermediate filament is connected to cell membrane or microtubules and microfilaments, while the other end is attached to nuclear membrane and connected to nuclear skeleton through a nuclear pore. Therefore, a complete structural supporting system is formed, which has a fixation effect on the nucleus and allows it to occupy certain space within the cell. In the process of mitosis, the intermediate filaments experience depolymerization and remodeling, envelope the spindle, and conduct a spatial orientation of the spindle and the chromosome. In addition, intermediate filaments collaborate with microtubules to participate in the transportation of intracellular materials. 


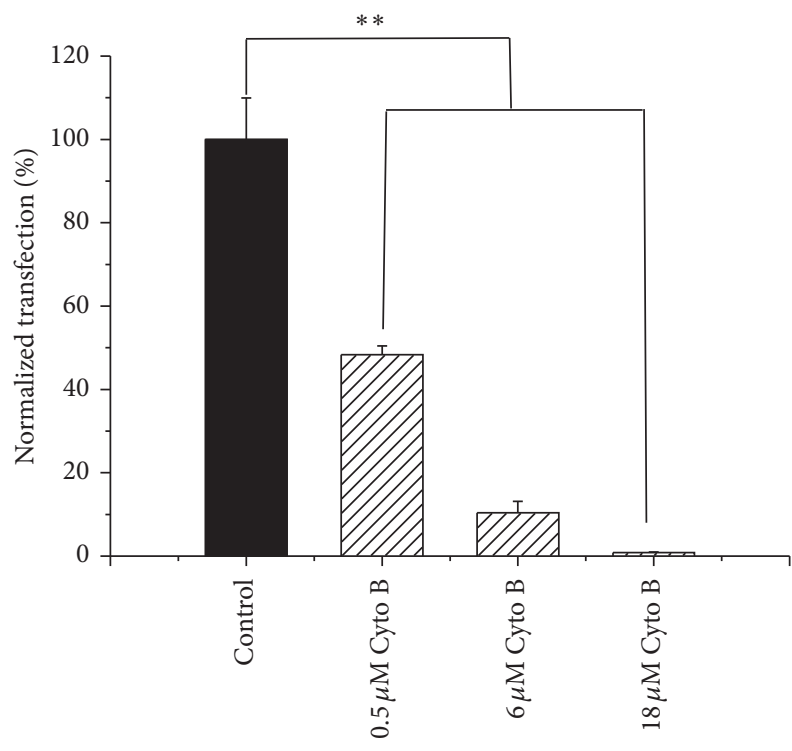

FIGURE 9: Effect of cytochalasin B (Cyto B) on transfection efficiency of P123-PEI-R13/DNA complexes in Hela cells at w/w ratio of 30. Cyto B was used as the inhibitors of microfilament. The RLU/mg protein in control cells was set to $100 \%$. Each data point represents the mean \pm standard deviation $\left(n=3,{ }^{* *} P<0.01\right)$.

Therefore, intermediate filaments are also an important part of intracellular transport of complexes, whose process will be greatly restricted without the support and assistance of intermediate filaments. It can be seen from Figure 10 that, after the treatment with acrylamide that has an inhibitory effect on skeleton structure of intermediate fibers, the transfection efficiency of complexes in Hela cell significantly decreased and the inhibition rate was up to $82 \%$. Thus it can be seen that intermediate filaments have a significant influence on P123PEI-R13 transfection.

\subsubsection{Effect of Cytoplasmic Dynein Inhibitors on Intracellular} Transport of P123-PEI-R13/DNA Complexes. The side of a microtubule close to the plasma membranes is the "+" end, while the side near microtubule organizing center of nuclear membranes is the "-" end. Microtubules are distributed in the cells in a radiative way toward the periphery. Studies have indicated that microtubules are related to intracellular transport of membrane vesicles and organelles, providing both a path for the transport of materials and a guiding role in its direction. The driving force for transport comes from two types of motor proteins. Materials in cells are driven by the dynein to move from "+" to "-" while kinesin has the opposite effect.

The complexes are transferred to the "-" end of intracellular canaliculus, namely, near-nucleus end, with the power source mainly from dynein. Therefore, this experiment chose sodium orthovanadate ( $\mathrm{SOV}$ ) to treat cells and study the influence of dynein on the transfection efficiency of P123PEI-R13. From Figure 10, with the increase of the inhibitor concentration, the inhibitory effect gradually increased. The inhibition rate was approximately $90 \%$ when the sodium

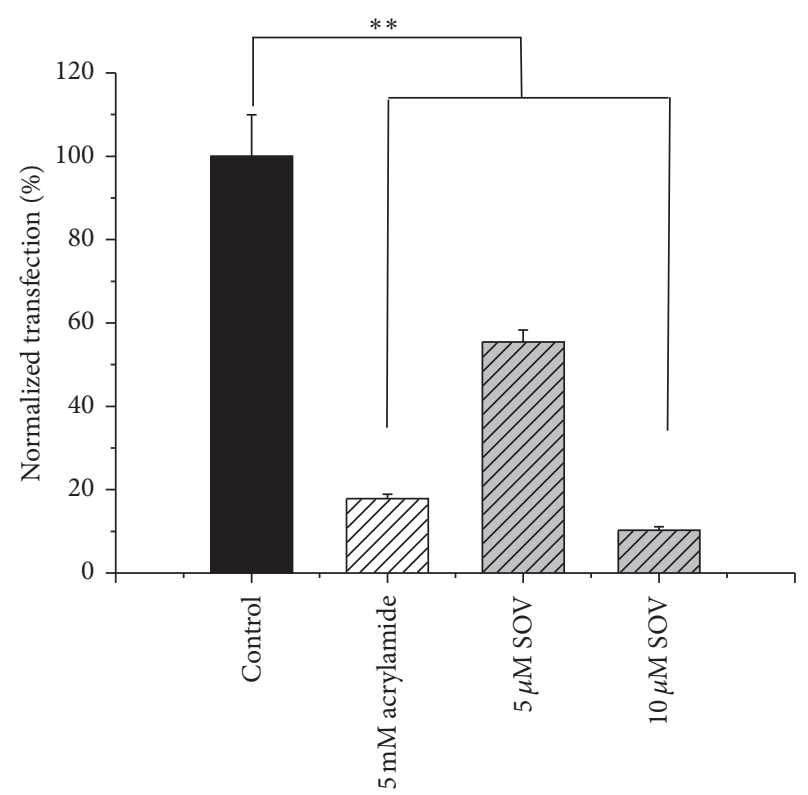

FIGURE 10: Effect of acrylamide and sodium orthovanadate (SOV) on transfection efficiency of P123-PEI-R13/DNA complexes in Hela cells at w/w ratio of 30 . Acrylamide was used as the inhibitors of intermediate filament (IF). SOV was used as the inhibitors of cytoplasmic dynein. The RLU/mg protein in control cells was set to $100 \%$. Each data point represents the mean \pm standard deviation $\left(n=3,{ }^{* *} P<0.01\right)$.

orthovanadate (SOV) concentration was $10 \mu \mathrm{M}$. According to the above results, dynein provides complexes with motivation to be transported toward the "-" end, namely, the cell nucleus within the cells. After the dynein was inhibited by sodium orthovanadate, its capability to carry the complexes was weakened, which significantly decreased the transfection efficiency.

\section{Conclusion}

In this paper, the binding affinity, cellular uptake, and subsequent intracellular trafficking of the nano-gene vector P123-PEI-R13 were investigated. The bifunctional peptide R13 had the ability of binding to $\alpha \mathrm{v} \beta 3$ positive cells in vitro. The modification of P123-PEI-R13 with R13 made it display a new property of internalization. P123-PEI-R13/DNA complexes were mainly endocytosed by clathrin-mediated endocytosis, caveolin-mediated endocytosis, and macropinocytosis. These three kinds of classical endocytic pathways simultaneously contributed to efficient gene delivery, which was in accordance with other researchers $[18,19]$. Macropinocytosis has attracted more attentions as a medicine and gene input pathway. There were reports that the endocytosis of TAT peptides and its cargo was achieved by macropinocytosis. It could be a more efficient endocytic pathway for the transfection process. In addition, there was a possible energy-independent route during the internalization for the modification of the cellpenetrating peptide TAT (49-57). 
After internalization, P123-PEI-R13/DNA complexes could escape from the endosome-lysosome system because of the protonation caused by its acidification and further took microtubule as the track and dynein as the dynamic source to be transported toward the microtubule (-) end, to wit nucleus, under the action of microfilament, and with the aid of intermediate filament.

\section{Competing Interests}

The authors declare that they have no competing interests.

\section{Acknowledgments}

This work was supported by National Natural Science Foundation of China under Grants nos. 81001024 and 81572989; Scientific Research Innovation Project of the Shanghai Municipal Education Commission under Grant no. 13 YZ097.

\section{References}

[1] K. H. Liu, X. Y. Wang, W. Fan et al., "Degradable polyethylenimine derivate coupled to a bifunctional peptide R13 as a new gene-delivery vector," International Journal of Nanomedicine, vol. 7, pp. 1149-1162, 2012.

[2] C. Vaquette, S. Fawzi-Grancher, P. Lavalle et al., "In vitro biocompatibility of different polyester membranes," Bio-Medical Materials and Engineering, vol. 16, no. 4, pp. S131-S136, 2006.

[3] S. D. Conner and S. L. Schmid, "Regulated portals of entry into the cell," Nature, vol. 422, no. 6927, pp. 37-44, 2003.

[4] M. Torres and T. D. Coates, "Function of the cytoskeleton in human neutrophils and methods for evaluation," Journal of Immunological Methods, vol. 232, no. 1-2, pp. 89-109, 1999.

[5] V. Rai, N. Dayan, and B. Michniak-Kohn, "A comparative evaluation of photo-toxic effect of fractionated melanin and chlorpromazine hydrochloride on human (dermal fibroblasts and epidermal keratinocytes) and mouse cell line/s (fibroblast Balb/c 3T3)," Toxicology in Vitro, vol. 25, no. 2, pp. 538-544, 2011.

[6] A. I. Ivanov, "Pharmacological inhibition of endocytic pathways: is it specific enough to be useful?" Methods in Molecular Biology, vol. 440, pp. 15-33, 2008.

[7] Z. Yu, Q. Xu, C. Dong et al., "Self-assembling peptide nanofibrous hydrogel as a versatile drug delivery platform," Current Pharmaceutical Design, vol. 21, no. 29, pp. 4342-4354, 2015.

[8] R. Peltier, G. Chen, H. Lei et al., "The rational design of a peptide-based hydrogel responsive to $\mathrm{H}_{2} \mathrm{~S}$," Chemical Communications, vol. 51, no. 97, pp. 17273-17276, 2015.

[9] M. Walsh, M. Tangney, M. J. O’Neill et al., "Evaluation of cellular uptake and gene transfer efficiency of pegylated poly-Llysine compacted DNA: implications for cancer gene therapy," Molecular Pharmaceutics, vol. 3, no. 6, pp. 644-653, 2006.

[10] G. Misinzo, P. L. Delputte, and H. J. Nauwynck, "Inhibition of endosome-lysosome system acidification enhances porcine circovirus 2 infection of porcine epithelial cells," Journal of Virology, vol. 82, no. 3, pp. 1128-1135, 2008.

[11] D. C. Lovelady, J. Friedman, S. Patel, D. A. Rabson, and C.M. Lo, "Detecting effects of low levels of cytochalasin B in 3T3 fibroblast cultures by analysis of electrical noise obtained from cellular micromotion," Biosensors and Bioelectronics, vol. 24, no. 7, pp. 2250-2254, 2009.

[12] Y. X. Chen, J. L. Li, Q. Q. Li et al., "Enhanced water-solubility, antibacterial activity and biocompatibility upon introducing sulfobetaine and quaternary ammonium to chitosan," Carbohydrate Polymers, vol. 143, pp. 246-253, 2016.

[13] Y. Solak, H. Atalay, I. Polat, and Z. Biyik, "Colchicine treatment in autosomal dominant polycystic kidney disease: many points in common," Medical Hypotheses, vol. 74, no. 2, pp. 314-317, 2010.

[14] J. S. Wadia, R. V. Stan, and S. F. Dowdy, "Transducible TAT-HA fusogenic peptide enhances escape of TAT-fusion proteins after lipid raft macropinocytosis," Nature Medicine, vol. 10, no. 3, pp. 310-315, 2004.

[15] P. E. G. Thorén, D. Persson, P. Isakson, M. Goksör, A. Önfelt, and B. Nordén, "Uptake of analogs of penetratin, Tat(48-60) and oligoarginine in live cells," Biochemical and Biophysical Research Communications, vol. 307, no. 1, pp. 100-107, 2003.

[16] Y.-X. Sun, W. Xiao, S.-X. Cheng, X.-Z. Zhang, and R.-X. Zhuo, "Synthesis of (Dex-HMDI)-g-PEIs as effective and low cytotoxic nonviral gene vectors," Journal of Controlled Release, vol. 128, no. 2, pp. 171-178, 2008.

[17] M. Arrio-Dupont, G. Foucault, M. Vacher, P. F. Devaux, and S. Cribier, "Translational diffusion of globular proteins in the cytoplasm of cultured muscle cells," Biophysical Journal, vol. 78, no. 2, pp. 901-907, 2000.

[18] L. Bromberg, V. Y. Alakhov, and T. A. Hatton, "Self-assembling Pluronic ${ }^{\circledR}$-modified polycations in gene delivery," Current Opinion in Colloid \& Interface Science, vol. 11, no. 4, pp. 217-223, 2006.

[19] I. M. Kaplan, J. S. Wadia, and S. F. Dowdy, "Cationic TAT peptide transduction domain enters cells by macropinocytosis," Journal of Controlled Release, vol. 102, no. 1, pp. 247-253, 2005. 

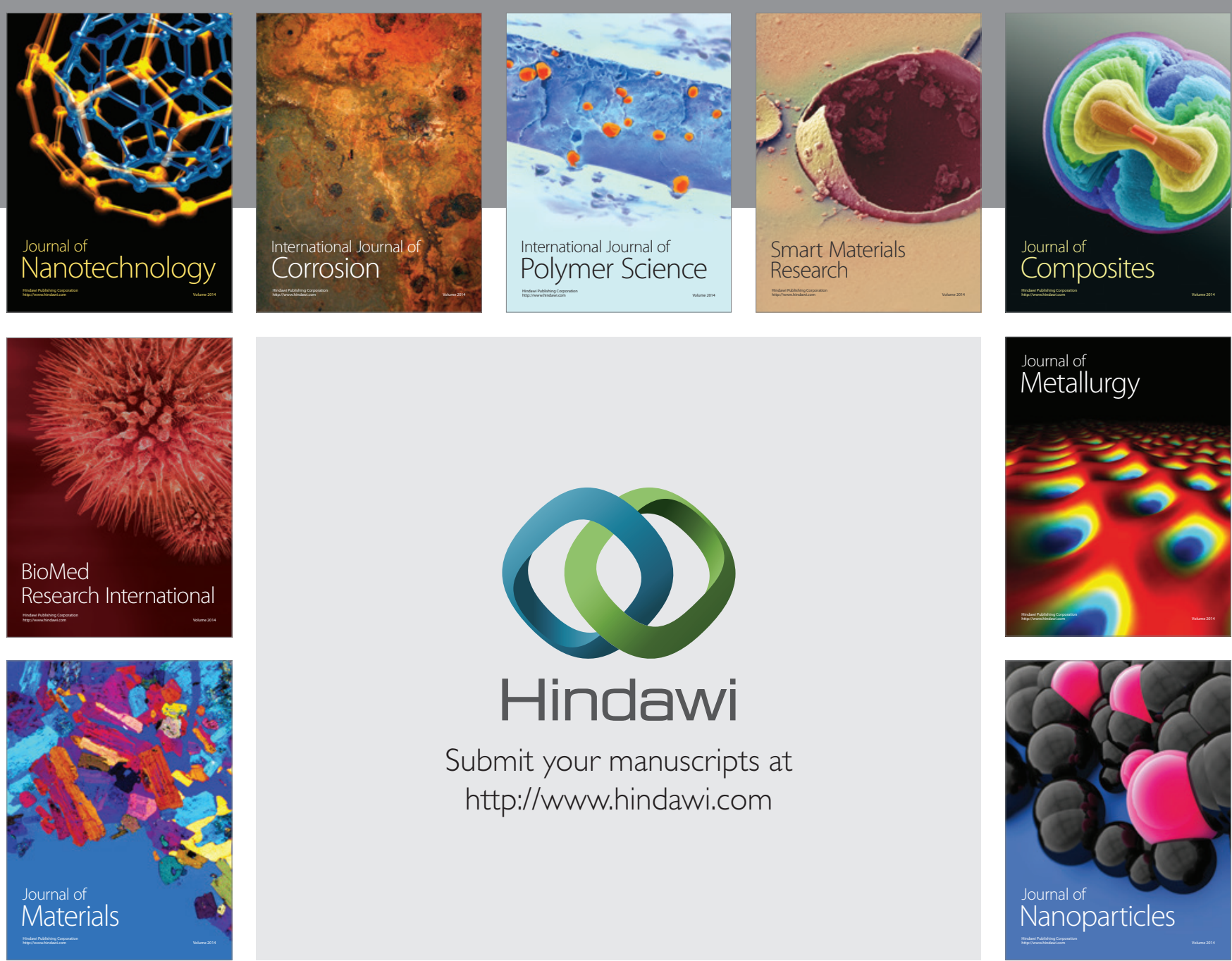

\section{Hindawi}

Submit your manuscripts at

http://www.hindawi.com

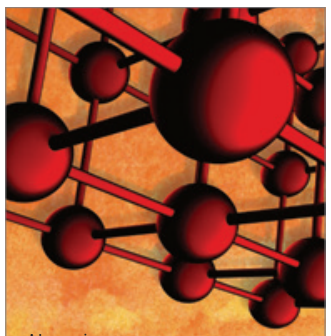

Materials Science and Engineering
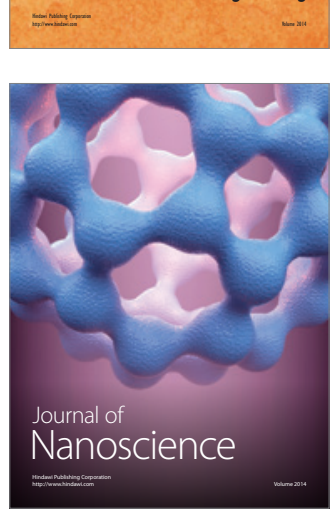
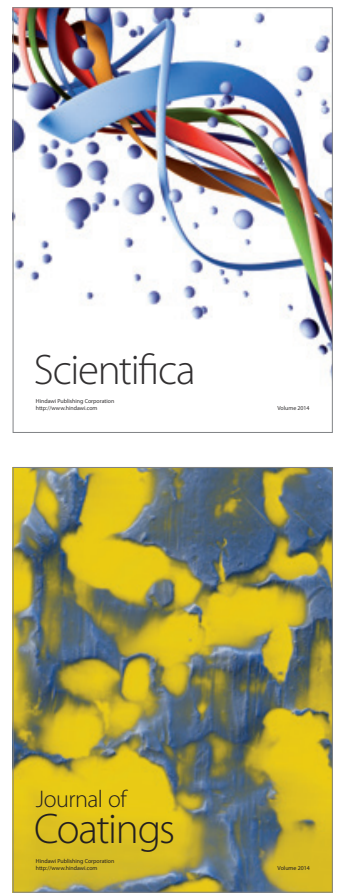
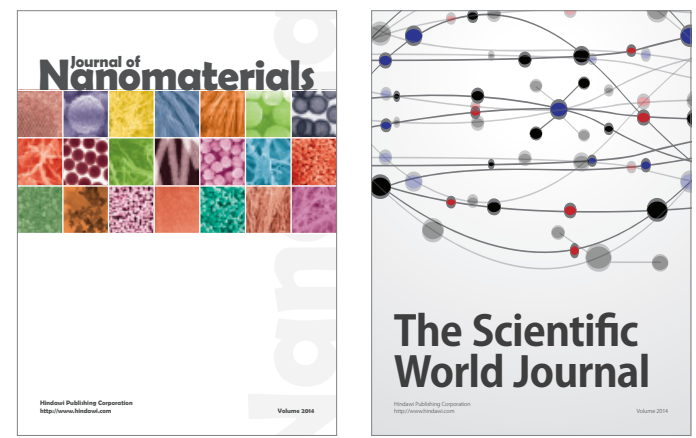

The Scientific World Journal
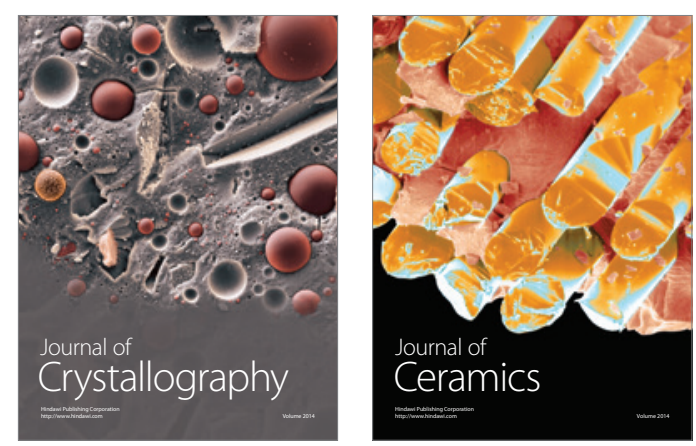
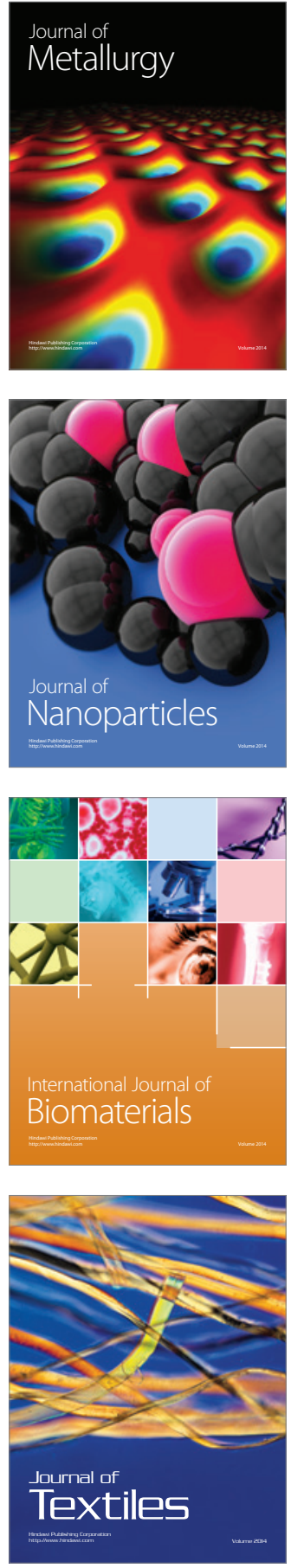